\title{
Further insight into AE37 peptide vaccination in prostate cancer
}

First draft submitted: 13 January 2017; Accepted for publication: 24 February 2017; Published online: 11 May 2017

Keywords: cancer vaccines $\bullet$ clinical trials $\bullet$ immunotherapy $\bullet$ patient selection $\bullet$ pre-existing immunity $\bullet$ prostate cancer

Cancer peptide vaccination, as an immunotherapeutic approach against solid tumors, is currently employed in several clinical research protocols. The underlying mechanism of peptide-based vaccines involves the generation of a T-cell immune response against tumor or enhancement of an endogenous antitumor immunity pre-existing in the host [1]. Although the rationale of cancer vaccination studies seems to be promising, therapeutic efficacy is rather limited [2,3]. One reason that could account for that is the inappropriate clinical trial design and patient selection, rather than the vaccine itself [3]. It is important to underline that the antitumor immune response is influenced by the respective immunological status and tumor-cell characteristics, thus implying heterogeneity among patients [4]. In respect to this, T-cell responses against certain peptides, including those derived from tumors, are mediated by specific human leukocyte antigen (HLA) molecules, therefore, the HLA polymorphism is another factor reflecting this variation in patients' immunological response [5]. Given that, patients' selection in vaccination studies requires careful consideration, so as to achieve therapeutic efficacy.

According to recent reports, pre-existing host immunity is essential in order to gain a therapeutic benefit, in the context of peptide cancer vaccines [2,6]. More specifically, patients with a pre-existing immunity to the vaccine antigen can develop fast and robust immune responses, upon vaccination. In addition, since pre-existing immunity against peptides integrated in the vaccine formulation, has been shown to be a predictive biomarker of response and therapeutic benefit, it should be used to select patients who are likely to respond to the vaccine [7]. On the contrary, delayed and insufficient immune responses are observed in patients with no immunological memory to the vaccine antigen and especially those with advanced cancer, characterized also by high immunosuppresion levels due to disease progression [8].

Considering the heterogeneity among individuals along with the role of pre-existing immunity prior to vaccination, a more personalized approach in peptide-based vaccination studies could result in better responses and further contribute to the development of effective cancer vaccines. In line with this, the HLA phenotype and levels of pre-existing immunity could be employed in clinical trials in order to identify cancer patients that could benefit from a specific peptide vaccine. In this respect, a recent study from our group showed that prostate cancer patients vaccinated with the Ii-key hybrid HER2 peptide [9], exhibit longer progression-free survival when they have increased levels of pre-existing immunity to the respective native HER2 peptide (AE36). In this study, additionally to enzyme-linked immunosorbent spot IFN- $\gamma$, we used the local reaction (LR) at the inoculation site of the first vaccine $(500 \mu \mathrm{g}$ peptide $+125 \mu \mathrm{g}$ granulocyte-macrophage colony stimulating factor) (LR1) as an approach for assessing pre-existing immunity, besides the generally

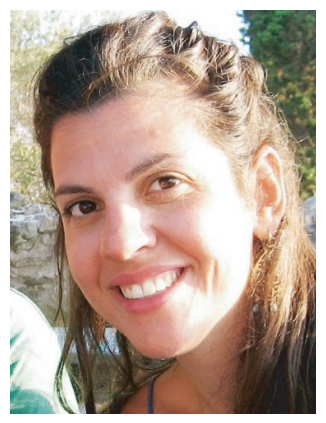

Eleftheria A Anastasopoulou Cancer Immunology \& Immunotherapy Center, Saint Savas Cancer Hospital, Athens, Greece

Tel.: +30 2106409496;

e.a.anastasopoulou@gmail.com

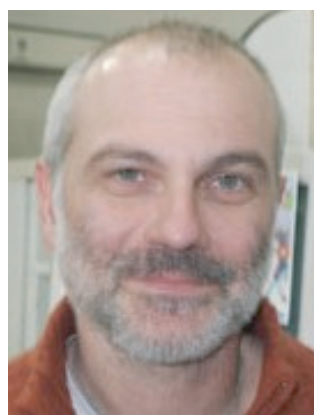

loannis F Voutsas

Cancer Immunology \& Immunotherapy Center, Saint Savas Cancer Hospital, Athens, Greece 
accredited immunomonitoring method of delayed type hypersensitivity (DTH) [10]. In particular, in standard DTH reactions (100 $\mu \mathrm{g}$ AE36 or AE37 without granulocyte-macrophage colony stimulating factor) used in the protocol prevaccination, was not found to be sufficient to evaluate pre-existing immunity for HER2, given the low concentration of peptide and the absence of immunoadjuvant, to trigger a usually low immunological memory response to self-antigens. Therefore, we considered LR1 as a more appropriate method to evaluate HER2 pre-existing immunity, in the context of immunomonitoring, which is also in agreement with previous reports describing that LR to the vaccination site can be used to evaluate immune responses [11].

Bearing in mind that AE37 is actually a multiepitope vaccine, potent to stimulate both $\mathrm{CD}^{+}$and $\mathrm{CD}^{+} \mathrm{T}$ cells, aiming at the induction of a generalized immune response [1], we decided to investigate the levels of pre-existing immunity to the vaccine. Most importantly, given the fact that the epitope-spreading effect is a significant parameter usually assessed in the context of vaccine evaluation, we investigated specific immunity to MHC class I restricted epitopes not integrated in the vaccine formulation, using specific multimers (dextramers) [12]. In particular, we assessed HLA-A2 and $-\mathrm{A} 24^{+}$patients, since these were the most commonly expressed alleles in our study group. More specifically, we investigated specific CD8 T cells against tumor-associated epitopes derived from HER2

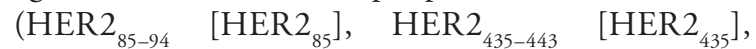
HER2 $\left.{ }_{369-377}[\mathrm{E} 75]\right), \quad$ PSA (PSA $_{146-154}\left[\mathrm{PSA}_{146}\right]$, PSA $_{153-161}\left[\right.$ PSA $\left._{153}\right]$ ), hTERT (hTERT ${ }_{540-548}$ ), PSMA (PSMA $_{27-35}$ ) and survivin (survivin M2 $2_{96-104}\left[\mathrm{SURV}_{96}\right]$, survivin $\left._{20-28}\left[\mathrm{SURV}_{20}\right]\right)$. Our findings showed that AE37 vaccinations boost specific antitumor immune responses against epitopes not integrated in the vaccine formulation, further implying an epitope-spreading effect. Namely, we detected relatively high frequencies of specific $\mathrm{CD} 8^{+} \mathrm{T}$ cell pre-existing immunity against PSA $_{153-161}$, an HLA-A24-restricted epitope, which was further enhanced upon vaccination and was associated with favorable clinical outcome in the respective HLA carriers. Moreover, we detected high pre-existing frequencies of specific $\mathrm{CD}^{+}{ }^{+} \mathrm{T}$ cells for E75 (HER2 ${ }_{369-377}$ ) and PSA $_{146-151}$ in HLA-A2 ${ }^{+}$patients that were also enhanced upon AE37 vaccination. However, specific E75 $\mathrm{CD}^{+}{ }^{+}$T-cell immunity in HLA-A2 ${ }^{+}$patients, either pre-existing or AE37 induced, seems to correlate with an unfavorable clinical outcome.

The immunological status of each patient along with the shifting tumor profile might explain why cytotoxic T lymphocyte E75-specific immunity failed to be interpreted into an effective clinical response. It is worth mentioning that under the pressure of the immune system, tumors alter the expression of HLA class I molecules, from total loss to reduced expression, a common escape mechanism that allows the selective growth of less immunogenic tumor variants during immunoediting [13]. In line with this, it has been previously described that high HER 2 expressing tumors are characterized by impaired antigen presentation, resulting in poor recognition of the respective tumor antigen by specific tumor-reactive $\mathrm{CD}^{+} \mathrm{T}$ cells [14]. Beside this, preclinical studies have noted the role of HER2 in the downregulation of HLA class I molecules, while results from clinical trials suggest that E75 vaccination might not favor breast cancer patients with tumors expressing high levels of HER2 [15]. In support to our results, a recent study by Tran et al. [16] reported that E75-based peptide vaccination can inhibit tumor growth only in low HER2 expressing tumors in HLA-A2+ transgenic mice. This and the fact that HER2 expression is known to increase significantly following disease progression in prostate cancer [17], could account for the inability of E75-specific cytotoxic T lymphocytes, detected in our patients, to exert an effective antitumor immune response with direct clinical impact. In other words, this potential downregulation of the HLA-A2 in high HER2 expressing tumors, could explain the unfavorable clinical outcome (shorter progression-free survival) observed in our patients exhibiting high preexisting immunity against E75 or with induced E75 immunity upon vaccination with AE37.

To conclude, our data, although hypothesis generating, might pave the way to unravel the prognostic/predictive significance of pre-existing antitumor immunological memory and how it can be employed so as to improve immunotherapeutic strategies. Recent cancer vaccination modalities are adopting a more personalized approach since they are based on the identification of patient's tumor mutanome and the prediction of potential neoepitopes that could represent attractive tumor antigens [18]. It is noteworthy to mention that this personalized approach is also reflected by the development of personalized peptide vaccines (PPV), which may include a maximum of four HLA-matched peptides [2], based on the pre-existing host immunity before vaccination. Results from Phase I and II clinical trials, have shown that PPV can induce specific immune responses with promising clinical outcomes in castrateresistant prostate cancer patients [19]. Hence, considering that in the last few years several clinical studies have employed PPV for the treatment of the prostate cancer in HLA-A24+ $4^{+}$and $-\mathrm{A} 2^{+}$patients $[2,19,20]$, our findings could further contribute to the development of the respective immunotherapeutic approach. In this respect, future immunotherapeutic protocols with the AE37 vaccine, could possibly achieve better clinical responses by recruiting patients with a pre-existing 
immunity to the native peptide. Regarding patient selection, we recommend that a DTH test with higher antigen concentration, with or without the immunoadjuvant, could possibly be used to successfully evaluate the levels of pre-existing immunity in patients prior to vaccination, along with the in vitro assessment of IFN- $\gamma$ enzyme-linked immunosorbent spot.

Considering all the above, it should be noted that employing $\mathrm{CD}^{+}{ }^{+} \mathrm{T}$-cell epitopes in peptide-based vaccines seems to be rather challenging, given the impaired expression of HLA class I molecules in tumor cells, thus research in this direction should remain viable.

\section{Financial \& competing interests disclosure}

The authors have no relevant affiliations or financial involvement with any organization or entity with a financial

\section{References}

Papers of special note have been highlighted as:

- of interest; $\bullet$ of considerable interest

1 Baxevanis CN, Papamichail M, Perez SA. Prostate cancer vaccines: the long road to clinical application. Cancer Immunol. Immunother. 64(4), 401-408 (2015).

2 Sakamoto S, Noguchi M, Yamada A, Itoh K, Sasada T. Prospect and progress of personalized peptide vaccinations for advanced cancers. Expert Opin. Biol. Ther. 16(5), 689-698 (2016).

-. A very informative review regarding personalized peptide-based vaccination.

3 Clifton GT, Kohrt HE, Peoples GE. Critical issues in cancer vaccine trial design. Vaccine 33(51), 7386-7392 (2015).

4 Schreiber RD, Old LJ, Smyth MJ. Cancer immunoediting: integrating immunity's roles in cancer suppression and promotion. Science 331(6024), 1565-1570 (2011).

5 Anastasopoulou EA, Voutsas IF, Keramitsoglou T et al. A pilot study in prostate cancer patients treated with the AE37 Ii-key-HER-2/neu polypeptide vaccine suggests that HLA-A*24 and HLA-DRB1*11 alleles may be prognostic and predictive biomarkers for clinical benefit. Cancer Immunol. Immunother. 64(9), 1123-1136 (2015).

6 Baxevanis CN, Perez SA. Cancer dormancy: a regulatory role for endogenous immunity in establishing and maintaining the tumor dormant state. Vaccines 3(3), 597-619 (2015).

7 Noguchi M, Sasada T, Itoh K. Personalized peptide vaccination: a new approach for advanced cancer as therapeutic cancer vaccine. Cancer Immunol. Immunother. 62(5), 919-929 (2013).

8 Pilla L, Rivoltini L, Patuzzo R, Marrari A, Valdagni R, Parmiani G. Multipeptide vaccination in cancer patients. Expert Opin. Biol. Ther. 9(8), 1043-1055 (2009).

9 Voutsas IF, Anastasopoulou EA, Tzonis P, Papamichail M, Perez SA, Baxevanis CN. Unraveling the role of preexisting immunity in prostate cancer patients vaccinated with a HER2/neu hybrid peptide. J. Immunother. Cancer 4, 75 (2016). interest in or financial conflict with the subject matter or materials discussed in the manuscript. This includes employment, consultancies, honoraria, stock ownership or options, expert testimony, grants or patents received or pending, or royalties.

No writing assistance was utilized in the production of this manuscript.

\section{Authors' contributions}

A Eleftheria wrote the commentary and $V$ Ioannis reviewed and edited the final version.

\section{Open access}

This work is licensed under the Creative Commons Attribution 4.0 License. To view a copy of this license, visit http://creativecommons.org/licenses/by/4.0/

10 Disis ML. Immunologic biomarkers as correlates of clinical response to cancer immunotherapy. Cancer Immunol. Immunother. 60(3), 433-442 (2011).

- Describes biomarkers of immunological and clinical response.

11 Rampling R, Peoples S, Mulholland PJ et al. A cancer research UK first time in human Phase I trial of IMA950 (novel multi peptide therapeutic vaccine) in patients with newly diagnosed glioblastoma. Clin. Cancer Res. 22(19), 4776-4785 (2016).

12 Mittendorf EA, Gurney JM, Storrer CE, Shriver CD, Ponniah S, Peoples GE. Vaccination with a HER2/neu peptide induces intra- and inter-antigenic epitope spreading in patients with early stage breast cancer. Surgery 139(3), 407-418 (2006).

13 Campoli M, Ferrone S. HLA antigen changes in malignant cells: epigenetic mechanisms and biologic significance. Oncogene 27(45), 5869-5885 (2008).

14 Mimura K, Ando T, Poschke I et al. T cell recognition of HLA-A2 restricted tumor antigens is impaired by the oncogene HER2. Int. J. Cancer 128(2), 390-401 (2011).

- HER2-overexpressing tumors may be more prone to escape from human leukocyte antigen A2 restricted cytotoxic T lymphocytes.

15 Mittendorf EA, Clifton GT, Holmes JP et al. Final report of the Phase I/II clinical trial of the E75 (nelipepimut-S) vaccine with booster inoculations to prevent disease recurrence in high-risk breast cancer patients. Ann. Oncol. 25(9), 1735-1742 (2014).

16 Tran T, Diniz MO, Dransart E et al. A therapeutic Her2/ neu vaccine targeting dendritic cells preferentially inhibits the growth of low Her2/neu-expressing tumor in HLA-A2 transgenic mice. Clin. Cancer Res. 22(16), 4133-4144 (2016).

17 Signoretti S, Montironi R, Manola J et al. Her-2-neu expression and progression toward androgen independence in human prostate cancer. J. Natl Cancer Inst. 92(23), 1918-1925 (2000). 
18 Carreno BM, Magrini V, Becker-Hapak M et al. Cancer immunotherapy. A dendritic cell vaccine increases the breadth and diversity of melanoma neoantigen-specific $\mathrm{T}$ cells. Science 348(6236), 803-808 (2015).

19 Sasada T, Yamada A, Noguchi M, Itoh K. Personalized peptide vaccine for treatment of advanced cancer. Curr. Med. Chem. 21(21), 2332-2345 (2014).
- Results from clinical trials of personalized peptide vaccines in prostate cancer.

20 Noguchi M, Itoh K, Yao A et al. Immunological evaluation of individualized peptide vaccination with a low dose of estramustine for HLA-A24+ HRPC patients. Prostate 63(1), $1-12(2005)$. 